This item was submitted to Loughborough's Research Repository by the author.

Items in Figshare are protected by copyright, with all rights reserved, unless otherwise indicated.

\title{
Sex differences in age-related changes on peripheral warm and cold innocuous thermal sensitivity
}

PLEASE CITE THE PUBLISHED VERSION

http://dx.doi.org/10.1016/j.physbeh.2016.05.045

\section{PUBLISHER}

(C) Elsevier

VERSION

AM (Accepted Manuscript)

\section{PUBLISHER STATEMENT}

This work is made available according to the conditions of the Creative Commons Attribution-NonCommercialNoDerivatives 4.0 International (CC BY-NC-ND 4.0) licence. Full details of this licence are available at: https://creativecommons.org/licenses/by-nc-nd/4.0/

\section{LICENCE}

CC BY-NC-ND 4.0

\section{REPOSITORY RECORD}

Inoue, Yoshimitsu, Nicola Gerrett, Tomoko Ichinose-Kuwahara, Yasue Umino, Saeko Kiuchi, Tatsuro Amano, Hiroyuki Ueda, George Havenith, and N. Kondo. 2019. "Sex Differences in Age-related Changes on Peripheral Warm and Cold Innocuous Thermal Sensitivity”. figshare. https://hdl.handle.net/2134/21637. 
\& Behavior

Elsevier Editorial System(tm) for Physiology

Manuscript Draft

Manuscript Number: PHB-D-16-00176R1

Title: Sex differences in age-related changes on peripheral warm and cold innocuous thermal sensitivity

Article Type: Research paper

Keywords: sex

ageing

thermal sensitivity

temperature

Regional differences

Corresponding Author: Dr Yoshimitsu Inoue, PhD

Corresponding Author's Institution: Osaka International University

First Author: Yoshimitsu Inoue, PhD

Order of Authors: Yoshimitsu Inoue, PhD; Nicola Gerrett, PhD; Tomoko Ichinose-Kuwahara; Yasue Umino; Saeko Kiuchi; Tatsuro Amano, PhD;

Hiroyuki Ueda, PhD; George Havenith, PhD; Narihiko Kondo, PhD

Abstract: Cutaneous thermal sensitivity to a warm and cold stimulus was compared among 12 older (OF, $65.2 \pm 1.0 \mathrm{yr}$ ) and 29 younger (YF, $21.6 \pm$ $0.2 \mathrm{yr}$ ) female participants, and 17 older (OM, $66.2 \pm 1.5 \mathrm{yr}$ ) and 13 younger ( $\mathrm{YM}, 21.2 \pm 0.4 \mathrm{yr})$ male participants to examine the effects of aging and sex. In a neutral condition $\left(27.5^{\circ} \mathrm{C}, 50 \% \mathrm{RH}\right)$ during rest, warm and cold thermal sensitivity was measured on eight body regions (forehead, chest, back, forearm, hand, thigh, calf, and foot). Using the method of limits, a thermal stimulator was applied to the skin at an adapting temperature and either increased or decreased at a constant rate $\left(0.3^{\circ} \mathrm{C} / \mathrm{s}\right)$ until the participants detected the temperature with a push button. Thermal sensitivity declined with ageing to both a cold (older: $1468.6 \pm 744.7 \mathrm{~W} / \mathrm{m} 2$, younger: 869.8 $\pm 654.7 \mathrm{~W} / \mathrm{m} 2, \mathrm{p}<0.001$ ) and warm (older: $2127.0 \pm 1208.3 \mathrm{~W} / \mathrm{m} 2$, younger: $1301.7 \pm 1055.2 \mathrm{~W} / \mathrm{m} 2, \mathrm{p}<0.001$ ) innocuous stimulus. YF and OF were more sensitive than YM and OM to both a warm and cold stimulus $(p<0.05)$. There was no interaction between age and sex suggesting that whilst thermal sensitivity decreases with age the decrease is similar between the sexes $(p>0.05)$. There was an interaction between temperatures, age and location and it seemed that cold thermal sensitivity was more homogenous for young and older participants however warm thermal sensitivity was more heterogeneous especially in the younger participants $(\mathrm{p}<0.05)$. Although the pattern was not similar between ages or sexes it was evident that the forehead was the most sensitive region to a warm and cold stimulus. Interestingly the decline in sensitivity observed with ageing occurred for all locations but was attenuated at the forehead in both males and females ( $p>0.05)$. 


\section{Yoshi Inoue PhD}

Laboratory for Human Performance Research

Osaka International University

Moriguchi

Osaka

570-8555

Japan

Dear Sir, Madam,

Please find attached a full length article for submission to the journal Physiology \& Behavior

The authors declare that they have no conflict of interest.

All experimental procedures were approved by the Osaka International University institutional ethics committee.

The authors declare the manuscript submitted to the Journal has not been published elsewhere or is not being considered for publication elsewhere and that the research reported will not be submitted for publication elsewhere until a final decision has been made as to its acceptability by the Journal. We look forward to hearing your response.

Kind regards

Yoshi Inoue 
We wish to thank the reviewers for taking time to provide very valuable and constructive comments on our manuscript. We have addressed each point and indicated where in the manuscript the changes have been made. We apologies in advance if we have mis-understood the points being made, please correct us if we are wrong. In some instances we have attempted to justify why changes have not been made and we are happy to discuss these points further if needed. After addressing the reviewers comments we have checked the manuscript again and have made some minor changes (e.g. changing 'subjects' to 'participants' to ensure consistency throughout). These are all minor changes to words to correct small errors (e.g. 'gender' to 'sex'), spelling, grammar etc. We have used track changes so these can be seen clearly.

Reviewer \#1: Sex differences in age-related changes on peripheral warm and cold innocuous thermal sensitivity.

Major Comments:

1. The authors use the Games-Howell post-hoc tests due to the unequal sample sizes. They, however, need to justify use of the ANOVA. If the assumption of homogeneity of variance is broken (due to the drastically different sample sizes) they need to use a non-parametric test.

Thank you for highlighting this. The Games-Howell is also used in circumstances of unequal variances. However, we doubled checked our data and noticed that in some (not all instances) homogeneity of variance was violated. We were able to restore the variance by a log transformation and have now included this information in the method sections of the data analysis (see page 9). As a result of this there were some alterations to the statistical findings, which have also been amended. These alterations did not impact the main interpretation of our findings.

We have also included more information in the data analysis section in the methods about the pre-analysis procedures to aid clarity.

2. Does skin fold thickness influence thermal sensing? It would seem like a strong possibility given the method the authors use to induce temperature change. If so the authors need to control for the difference between the sexes and ages.

Both reviewers have commented on this point and we have discussed it extensively in response to reviewer 2. Please see below.

3. Figure 1: I do not believe it is statistically justifiable to graph data as a 3 way ANOVA (location $X$ sex $X$ age) but then include results from both said 3 way ANOVA and separate 2 way ANOVAs (location X age). Is there much information even gained form including location information in the ANOVA? Although the authors provide differences in temperature detection by location (Table 3), doesn't a lot of this have to do with the size of the receptive field (which was no accounted for)? 
We apologies that the information presented in Figure 1 and the corresponding legend was from pervious analysis and we had not updated it. The Figure and legend has been updated to correspond with the post hoc analysis from the three-way ANOVA's for cold sensitivity and warm sensitivity data.

The authors of the manuscript have been interested in regional differences in thermal sensitivity (perceptual and autonomic) for some time and have published this data over the last 20 years. Whilst it may appear that not much information is gained from including the location data into the analysis as we only gained information about facial sensitivity, we believe that this, in itself is important. Thermophysiological papers, whether they be autonomic thermoregulation studies or behavioural/perceptual studies are often done on one location and sometimes generalised for the entire body. However, there has been in increase more recently in the awareness of this issue and more papers are being published to address it (Gerrett et al. 2014; Smith et al. 2013; Smith \& Havenith 2012 etc). Therefore we wish for the location data to be included in the analysis and a theme of this paper.

The technique we have used to assess regional differences is a popular method of choice for regional thermal sensitivity studies (Lee et al. 2012; Gerrett et al. 2014; Takeda et al. 2016; Ouzzahra et al. 2012 etc) as it is difficult to produce stimulation equipment that will be useable on different people with different anthropometric characteristics. There are many options for assessing regional sensitivity but it is difficult to decide which one is more appropriate; do you ensure that all areas are stimulated with the same surface area or one that is proportional to its size or proportional to the number of potential receptive units in the area (which is currently unknown and will vary from person to person). Typically the size of the stimulation is identical across different regions as a method of control. Interestingly thought, a number of studies suggest that the perpetual responses cannot be solely explained by the density or properties of the peripheral thermoreceptors as the hands, an area known to be densely populated did not produce the largest thermal sensitivity response (Gerrett et al. 2014; Gerrett et al. 2015). It has been suggested by others (Nakamura et al. 2008; Cotter \& Taylor 2005) who have also noted that the perceptual response cannot be solely explained by the density or properties of peripheral thermoreceptors and that the central nervous system must assign a weighing factor to areas of physiological importance such as the face and torso. However this still required further support.

\section{Minor:}

1. In the Abstract the authors should define the methods of limits test.

This information has now been included.

\section{Reviewer \#2:}

The authors present an original study that is interesting and relevant to the field of this Journal. The manuscript is very well written and presented clearly. The following should be considered by the authors to improve the strength of this 


\section{manuscript:}

In their analyses and discussion of observed sex differences in regional thermal sensitivity, the authors should give consideration to the subcutaneous fat differences observed between male and female subjects. The authors observed the lowest baseline skin temperature in the older females. This group also had significantly higher subcutaneous fat than all other groups, as measured by the sum of 7 skinfold measurements. As noted by other investigators, adiposity significantly influences observed skin temperatures across various body regions and may influence regional thermal sensitivity. Therefore, it is important for the authors to acknowledge that the sex (and age amongst females) differences in subcutaneous fat measures may, at least in part, be contributing to observed differences in the regional thermal sensitivities. The investigators should consider additional analyses accounting for subcutaneous fat differences.

Both reviewer 1 and 2 both raised a point regarding the role of subcutaneous fat on thermal sensitivity. Even though we disagree with this comment we realized that many readers may have a similar opinion therefore we have included a section to explain the minimal role subcutaneous fat can have on thermal sensitivity. We hope that this explanation justifies our position on this point but we would be happy to discuss it further if required.

Whilst body fat and/or skin fold thickness can impact autonomic thermoregulation there is limited evidence for its role in perceptual thermal sensitivity. As highlighted by reviewer 2 , it has consistently been reported that participants with more fat tend to have a lower skin temperature (LeBlanc 1954; Livingstone et a. 1989; Fro 1990; Claessens-van Ooijen et al. 2006). Our findings agree with this as our OF had a higher $\Sigma 7 \mathrm{SF}$ and a lower mean skin temperature, whilst our males demonstrated no differences in $\Sigma 7 \mathrm{SF}$ or skin temperature. However, there is very limited evidence to show that skin fold thickness or body fat affects thermal sensitivity. Budd et al. (1991) found that whilst fatter participants differed in skin temperature and core temperature they felt no less uncomfortable to a whole body cold exposure test compared to leaner individuals. This has been supported more recently by Fournet (2013) who found that despite colder skin temperatures, fatter participants had similar local thermal sensations as their leaner counterparts. Ouzzahra (2012) also found no relationship between thermal sensation and skin fold thickness across numerous sites on the body. We could not find any studies in the literature demonstrating an impact of subcutaneous fat on thermal sensitivity (regardless of measurement technique (whole body stimulus, method of limits, magnitude estimation etc).

There are a number of reasons that this has either not been investigated and/or no differences have been observed. Along with other sensory receptors, the thermoreceptors which are being stimulated are located in the dermis of the skin. Notably, cold and warm receptors have been found at different depths within the dermis and this may impact the latency of the thermoreceptors and affect the perceptual thermal sensation experienced (Zotterman, 1953; Guyton, 1991; Hensel \& Andres 1984; Hensel 1981). Epidermal thickness is almost constant over the body, except the palms of the hands and sole of the feet where the stratum corneum can vary but these sites were not investigated in this experiment. Subcutaneous fat on the other hand, sits below the dermis in the hypodermis where neither cold nor warm 
thermoreceptors lie. The reason autonomic response may be influenced by subcutaneous fat is that the sweat glands and cutaneous blood vessels are located in the hypodermis, which is the site of adipose tissue.

However, our methods do not accurately measure skin thickness as skin fold calipers combines both subcutaneous fat and skin thickness, with subcutaneous fat contributing more substantially to this measure. Out of interest we ran simple regression correlations between each site (skin fold) and the corresponding thermal sensitivities for warmth and cold. The correlation ranged between 0.004-0.1, p>0.05. We also ran simple regression between the sum of seven skin folds and each sites thermal sensitivity for warmth and cold and found similar results. Based on the scientific theory and the information from some basic analysis we do not believe that skin fold thickness or subcutaneous fat will influence thermal sensation hence why we do not wish to analyse the data with this as a cofounding variable. We do however recognize that this information may be of interest to the reader and as such we have included this on our revision (see pages 16-17). 


\section{*Highlights}

\section{Highlights:}

- Forehead thermal sensitivity is persevered with ageing

- Regional cold sensitivity is homogenous for the young and elderly

- Regional warm sensitivity is heterogeneous especially in the young

- Females are more sensitive to innocuous warm and cold thermal stimulation than males

- Ageing does not changed the sex related differences in thermal sensitivity 


\section{Sex differences in age-related changes on peripheral warm and cold innocuous thermal} sensitivity

Yoshimitsu Inoue $^{\mathrm{a}}$,Nicola Gerrett ${ }^{\mathrm{b}}$ Tomoko Ichinose-Kuwahara ${ }^{\mathrm{a}}$, Yasue Umino ${ }^{\mathrm{a}}$, Saeko Kiuchi $^{\mathrm{a}}$, Tatsuro Amano ${ }^{\mathrm{dc}}$, Hiroyuki Ueda $^{\mathrm{d} \epsilon}$, George Havenith $^{\mathrm{e}}$ and Narihiko Kondo ${ }^{\mathrm{d} \epsilon}$

${ }^{a}$ Laboratory for Human Performance Research, Osaka International University, Moriguchi, Osaka 570-8555, Japan, inoueyos@ oiu.jpinoueyos@hus.oiu.ac.jp

${ }^{\mathrm{b}}$ Institute of Sport and Exercise Science, University of Worcester, Henwick Road, Worcester, WR26AJ, United Kingdom. N.Gerrett@icloud.com

EOsaka Shin-ai College, Tsurumi-ku, Osaka 538-0053, Japan. Ueda@osaka-shinai.ac.jp

${ }^{c}$ Laboratory for Applied Human Physiology, Graduate School of Human Development and EnvrionmentEnvironment, Kobe University, Kobe 657-8501, Japan. Kondo@kobe-u.ac.jp ${ }^{\mathrm{d} O s a k a}$ Shin-ai College, Tsurumi-ku, Osaka 538-0053, Japan. Ueda@ osaka-shinai.ac.jp

${ }^{\mathrm{e}}$ Environmental Ergonomics Research Centre, Loughborough Design School, Loughborough University, Loughborough, LE113TU, United Kingdom. G.Havenith@1boro.ac.uk

\section{Address correspondence to:}

Corresponding author: Dr. Y. Inoue inoueyos@hus.oiu.ac.jp 


\section{Abstract}

$\underline{C} U$ sing the methods of limits, cutaneous thermal sensitivity to a warm and cold stimulus was compared among 12 older (OF, $65.2 \pm 1.0 \mathrm{yr}$ ) and 29 younger (YF, $21.6 \pm 0.2 \mathrm{yr}$ ) female participants, and 17 older $(\mathrm{OM}, 66.2 \pm 1.5 \mathrm{yr})$ and 13 younger $(\mathrm{YM}, 21.2 \pm 0.4 \mathrm{yr})$ male participants to examine the effects of aging and sex. In a neutral condition $\left(27.5^{\circ} \mathrm{C}, 50 \%\right.$ $\mathrm{RH})$ during rest, warm and cold thermal sensitivity was measured on eight body regions (forehead, chest, back, forearm, hand, thigh, calf, and foot)._u - Using the method of limits, a thermal stimulator was applied to the skin at an adapting temperature and either increased or decreased at a constant rate $\left(0.3^{\circ} \mathrm{C} / \mathrm{s}\right)$ until the participants detected the temperature with a push button. Thermal sensitivity declined with ageing to both a cold (older: $1468.6 \pm 744.7$ $\mathrm{W} / \mathrm{m}^{2}$, younger: $869.8 \pm 654.7 \mathrm{~W} / \mathrm{m}^{2}, \mathrm{p}<0.001$ ) and warm (older: $2127.0 \pm 1208.3 \mathrm{~W} / \mathrm{m}^{2}$, younger: $\left.1301.7 \pm 1055.2 \mathrm{~W} / \mathrm{m}^{2}, \mathrm{p}<0.001\right)$ innocuous stimulus. $\mathrm{YF}$ and OF were more sensitive than $\mathrm{YM}$ and $\mathrm{OM}$ to both a warm and cold stimulus $(\mathrm{p}<0.05)$. There was no interaction between age and sex suggesting that whilst thermal sensitivity decreases with age the decrease is similar between the sexes $(p>0.05)$. There was an interaction between temperatures, age and location and it seemed that cold thermal sensitivity was more homogenous for young and older participants however warm thermal sensitivity was more heterogeneous especially in the younger participants $(\mathrm{p}<0.05)$. Although the pattern was not similar between ages or sexes it was evident that the forehead was the most sensitive region 
to a warm and cold stimulus. Interestingly the decline in sensitivity observed with ageing occurred for all locations but was attenuated at the forehead in both males and females $(\mathrm{p}>0.05)$.

Key words: sex, ageing, thermal sensitivity, temperature, regional differences

Highlights:

- Forehead thermal sensitivity is persevered with ageing

- Regional cold sensitivity is homogenous for the young and elderly

- Regional warm sensitivity is heterogeneous especially in the young

- Females are more sensitive to innocuous warm and cold thermal stimulation than males

- Ageing does not changed the sex related differences in thermal sensitivity 


\subsection{Introduction}

Behavioral and autonomic temperature regulation is mediated by inputs from afferent signals located both centrally and peripherally. Deterioration in the afferent nerves, such as a decrease in number and density of myelinated and unmyelinated fibres, reductions in neurotransmitters and their host cells are typical age-related declines in neurological functioning [1]. Age-dependent neural degeneration observed in thermoreceptors can reduce both physiological and perceptual thermal sensitivity. Impaired cutaneous vascular function and delays in the sweating and shivering thresholds have been reported in age comparison studies $[2,3,4,5,6]$. Likewise, reduced thermal sensations in older adults have been reported, alongside a number of other tactile responses (touch, pressure and noxious thermal sensitivity) $[7,8,9,10,11]$. With an increasing ageing population and increased reports of climate change, understanding the age related changes in our ability to thermoregulate is vitally important. Perceptual thermal sensitivity provides immediate feedback about the thermal state of the body and initiates a set of desired actions to correct the thermal imbalance. As a result, the ability to sense temperature acts as our first line of defence against thermal imbalance yet relatively little is known about this in comparison to our physiological responses.

It has been suggested that thermal sensitivity declines with age although the findings have been contradictory. Kenshalo [12] assessed the thermal threshold of the thenar eminence and sole of the foot to either an increasing or decreasing temperature. He noted an age related decrement only at the foot to an increasing temperature. Harju et al. [13] reported no effect of age on cold or warm threshold detection at the thernar eminence; lateral upper arm, lateral knee or sole of the foot. However, Haung et al. [8] reported age related decrements to warm and cold thresholds on the dorsal hand and foot using the same technique as Kenshalo [12]. 
Experimental data on the age-related changes to thermal stimulation have been contradictory but recently there is evidence to suggest that the age-related decline in thermal sensitivity is site specific. Tochihara et al. [7] reported age related decrements in warm threshold detection at the hand, foot and shin but not at the chest, abdomen, upper arm, forearm or thigh. This non-uniform decline may have contributed to the lack of clarity over thermal sensitivity especially when studies investigated a limited number of locations. Typically, thermal sensitivity is limited to one or two locations across the body despite seminal research indicating regional distribution of thermoreceptors across the body [14, 15]. Whilst most studies investigate a limited number of body sites, recently extensive body maps to a warm and cold innocuous stimulus have recently been reported in males and females ( 20 years) $[16,17$, 18]. However, more information about regional thermal sensitivity in older individuals in comparison to younger adults is required.

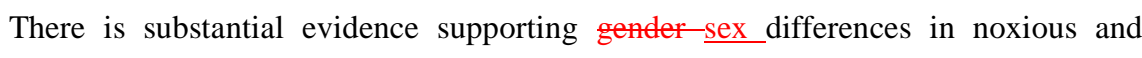
innocuous thermal sensitivity with females being more sensitive than males $[17,19,20,21]$. However this information is currently limited to relatively young adults ( 20-30 yrs). Overall, the literature suggests that thermal sensitivity may decline with age and females are more sensitive than males but whether the decline in thermal sensitivity with ageing is similar between males and females is unknown. Therefore the aim of the present study was to assess the effect of aging and sex on regional thermal sensitivity to a warm and cold stimulus. To address this we measured the threshold detection to an increasing and decreasing thermal stimulator on 8 locations across the body in older and younger males and females. 


\subsection{Methods}

\subsection{Participants}

Seventy-one participants volunteered for this study, of which 12 were older female (OF), 29 younger female (YF), 17 older male (OM) and 13 younger male (YM). The physiological characteristics of the participants are shown in Table 1. The young females were not taking oral contraceptives and were tested during the early- or mid-follicular phase (4-10 days after menstruation onset). Older female participants were all post-menopausal and were not undergoing hormonal replacement therapy. Participants completed health screen questionnaires and informed consent prior to taking part. Osaka International University institutional ethics committee for human investigations approved this study, which was carried out in accordance with the Declaration of Helsinki.

\subsection{Experimental design}

The aim of the investigation was to compare regional sensitivity to a hot and a cold stimulus in young and elderly males and females during rest. To achieve these aims an independent design was opted for, during which, regional thermal sensitivities to an increasing and decreasing thermal probe was investigated. A total of 8 regional body segments were chosen representing major anatomical landmarks (anterior and posterior torso and extremities). The testing sequence of the locations was randomized to reduce order effect. However, cold sensitivity test always preceded the warm sensitivity test. After completing both tests a 5 minute rest period was taken and the process repeated an additional 2 times to reduce unsystematic errors and order effects. Participants were familiarised with the test prior to the 
main experiment. The experiments were conducted between early August and late October in Japan.

\subsection{Experimental protocol}

Each participant completed a pre-test session for anthropometric measurements; including stature, weight and skin fold thickness. Body surface area (AD) was calculated using the following equation [22]:

$$
\operatorname{AD}\left(\mathrm{m}^{2}\right)=\text { Mass }(\mathrm{kg})^{0.444} \times \text { Height }(\mathrm{m})^{0.663} \times 88.83
$$

Skinfold thickness was measured using skinfold calipers over the chest, subscapular, suprailiac, triceps, anterior forearm, anterior thigh, and posterior calf and the sum of 7 skin folds calculated $(\Sigma 7 \mathrm{SF})$. They then completed a submaximal incremental exercise test on a cycle ergometer (Aerobike 75 XLII, Combi Corporation, Tokyo, Japan) for the estimation of maximal $\mathrm{O}_{2}$ uptake $\left(\dot{\mathrm{VO}}_{2} \max \right)$ based on the Åstrand Rhyming method. The test consisted of four progressive exercise stages each lasting 5 minutes. Heart rate (Polar Electro Oy, Kemple, Finland) was recorded during the last minute of each stage. Estimation of $\dot{\mathrm{VO}}_{2} \mathrm{max}$ was then calculated from the ACSM metabolic equation for cycling [23]. Participants were asked to wear a pedometer (JM-200, Yamasa, Tokyo, Japan) for one week to estimate typical physical activity (PA) levels (steps/day). Participants were instructed to wear the pedometer at the level of the hip throughout the day while following their normal daily routine.

On a separate day, participants visited the laboratory for the thermal sensitivity test. Participants were asked to refrain from strenuous exercise and alcohol the day before testing. To avoid any effects of diet-induced thermogenesis and for better control of hydration, no food or water was ingested from at least $1 \mathrm{~h}$ prior to arrival at the laboratory until the end of the test. 
Dressed in minimal attire (sports bra and shorts for females-subjects; shorts only for males), participants entered the environmental chamber (SRH-30VEVI2, Nagano Science, Japan) set at $27.5^{\circ} \mathrm{C}$ and the relative humidity $(\mathrm{RH})$ was $50 \%$. Whilst resting in a chair, skin and oral thermistors where applied (SZL64, Takara Thermistor Instruments, Yokohama, Japan). Four skin thermistors were applied to the chest, forearm, thigh, and calf (SZL64, Takara Thermistor Instruments, Yokohama, Japan). Core temperature was estimated using an oral thermometer $\left(\mathrm{T}_{\mathrm{or}}\right)$ placed under the tongue and values recorded after a 5-minute stabilization period. $\mathrm{T}_{\text {or }}$ and local skin temperatures were recorded at three times points; prior to testing and at the end of each 5-minute rest period between thermal sensitivity tests. Data was recorded onto a computer-controlled data logger (model K722; Takara Thermistor Instruments, Yokohama, Japan) and the three values averaged. Mean skin temperature $\left(\mathrm{T}_{\mathrm{sk}}\right)$ and mean body temperature $\left(\mathrm{T}_{\mathrm{b}}\right)$ were calculated using the following equations [24]:

$$
\begin{gathered}
\mathrm{T}_{\text {sk }}=0.3 \mathrm{~T}_{\text {chest }}+0.3 \mathrm{~T}_{\text {forearm }}+0.2 \mathrm{~T}_{\text {thigh }}+0.2 \mathrm{~T}_{\text {calf }} \\
\mathrm{T}_{\mathrm{b}}=0.9 \mathrm{~T}_{\text {or }}+0.1 \mathrm{~T}_{\mathrm{sk}}
\end{gathered}
$$

After a 30-minute stabilization period the thermal sensitivity test, using the method of limits was conducted. The test involved applying a hand held square (25 mm x $25 \mathrm{~mm}$ ) thermostimulator (Intercross-200, Intercross Co., Tokyo, Japan) to 8 locations on the body (forehead, chest, scapula, anterior forearm, dorsal hand, anterior thigh, posterior calf, and dorsal foot) in a randomized order with at least 1 minute separating locations.

The thermostimulator consisted of a heat-flux sensor, a peripheral temperature sensor and a thermode consisting of Peltier elements. Initially, the probe was placed on the skin and adjusted to the skin temperature. When the 2 temperatures were equal, the heat flux became 0 . The heat flux was maintained at $\pm 30 \mathrm{~W} / \mathrm{m}^{2}$ for 5 seconds before taking the measurements. The 
probe temperature was then increased or decreased by $0.3^{\circ} \mathrm{C} / \mathrm{s}$ until the participant felt a warm or cold sensation. Participants indicated when they detected a warm or cold sensation by pressing a hand held switch in their dominant hand. Thermal sensitivity is indicated as the difference between the heat flux at the commencement of the test and when the participant detects a temperature change. The difference in the case of the exoergic reaction was defined as the warm threshold, and in the case of the endoergic reaction was defined as the cold threshold. Warm and cold thresholds are expressed as absolute values $\left(\mathrm{W} / \mathrm{m}^{2}\right)$ and a lower heat flux value indicates a greater thermal sensitivity.

\subsection{Data analysis and statistics}

Warm and cold thresholds at each site were calculated as mean values of three thermal sensitivity tests. Statistical analysis was conducted using Statistical Package (SPSS) version 22.0. Differences in warm and cold thresholds across different body regions were analysed using three-way ANOVA with location as the independent variable and sex gender and age as between subject factors. All data was checked for any outliers, normality (Shapiro-Wilk), homogeneity of variance (Levenes' test) and sphericity. Warm and cold threshold data violated homogeneity of variance and were natural logarithm transformed for ANOVA tests. This resulted in all data having equal variance and normal distribution. When sphericity was violated Greenhouse-Geisser correction was used. Due to unequal sample sizes the Games-Howell post-hoc comparison test was used. Unless otherwise stated, all measurements are expressed as means with standard errorsdeviations $( \pm$ S.E.M) and significance is defined as $\mathrm{p}<0.05$. 


\subsection{Results}

In females, there were no age-related differences in mass, surface area-to-mass ratio and PA levels $-(\mathrm{p}>0.05)$ (see Table 1). Height and $\mathrm{VO}_{2} \max$ were lower in OF than $\mathrm{YF}(\mathrm{p}<0.05)$ and $\Sigma 7 \mathrm{SF}$ was greater in the OF group than YF $(\mathrm{p}<0.05)$. In males there were no age-related differences in mass, surface area-to-mass ratio, $\Sigma 7 \mathrm{SF}, \dot{\mathrm{VO}_{2}} \mathrm{max}$, and PA levels ( $>0.05$ ). However, OM were shorter than YM $(\mathrm{p}<0.05)$.

There were no gendersex-related differences in $\dot{\mathrm{VO}}_{2} \mathrm{max}$, PA levels or surface area to mass ratio $(p>0.05)$. Although males were taller and heavier than females $(p<0.05)$ but had a lower $\Sigma 7 \mathrm{SF}(\mathrm{p}<0.05)$.

During the thermal sensitivity tests, there were no age-related differences in $\mathrm{T}_{\text {or }}$ and $\mathrm{T}_{\mathrm{b}}$ in females $(\mathrm{p}>0.05)$. However, $\mathrm{T}_{\mathrm{sk}}$ was lower in OF compared to $\mathrm{YF}(\mathrm{p}<0.05)$ (Table 2). No age-related differences were observed in $\mathrm{T}_{\text {or }}, \mathrm{T}_{\mathrm{sk}}$, and $\mathrm{T}_{\mathrm{b}}$ in males (Table 2). There were no sexgender-related differences in $\mathrm{T}_{\mathrm{or}}, \mathrm{T}_{\mathrm{sk}}$, and $\mathrm{T}_{\mathrm{b}}(\mathrm{p}<0.05)$.

\subsection{Cold thermal sensitivity}

The absolute heat-flux thresholds for cold sensitivity of each body region in the four groups are shown in Figure 1. Three way-ANOVA revealed a main effect of age as the elderly group had a lower thermal sensitivity than the younger group $\left(1468.6 \pm 744.7 \mathrm{~W} / \mathrm{m}^{2}\right.$ and 869.8 $\pm 654.7 \mathrm{~W} / \mathrm{m}^{2}$, respectively, $\left.\mathrm{p}<0.001\right)$. A main effect of sex was also found as females had a higher thermal sensitivity in comparison to males $\left(546.3 \pm 200.3 \mathrm{~W} / \mathrm{m}^{2}\right.$ and $882.0 \pm 382.4$ $\mathrm{W} / \mathrm{m}^{2}$, respectively, $\left.\mathrm{p}<0.001\right)$. A main effect of location was observed and post hoc comparisons are presented in Table $3(\mathrm{p}<0.001)$. Inclusive of all ages and sex, high to low regional thermal sensitivity was in the following order: forehead $\left(688.2 \pm 333.7 \mathrm{~W} / \mathrm{m}^{2}\right)$, dorsal 
hand $\left(978.4 \pm 575.9 \mathrm{~W} / \mathrm{m}^{2}\right)$, scapula $\left(1074.8 \pm 631.7 \mathrm{~W} / \mathrm{m}^{2}\right)$, posterior thigh $(1100.1 \pm 545.9$ $\left.\mathrm{W} / \mathrm{m}^{2}\right)$, anterior forearm $\left(1113.3 \pm 619.1 \mathrm{~W} / \mathrm{m}^{2}\right)$, dorsal foot $\left(1209.8 \pm 732.9 \mathrm{~W} / \mathrm{m}^{2}\right)$, posterior calf $\left(1244.8 \pm 584.8 \mathrm{~W} / \mathrm{m}^{2}\right)$ and chest $\left(1253.3 \pm 662.0 \mathrm{~W} / \mathrm{m}^{2}\right)$. An interaction was observed between age and location and post hoc comparison revealed that all 8 body regions were more sensitive in the younger group compared to the elderly group $(\mathrm{p}<0.05)$. An interaction was also observed between sex and location and post hoc comparison revealed that all locations except the hand waswere more sensitive in females compared to males $(\mathrm{p}<0.05)$.

There was no interaction between age, sex and location $(\mathrm{p}>0.05)$ but when the effect of location and age was analysed for males and females separately, the results revealed an interaction between age and location. Post hoc comparison indicated that thermal sensitivity was different in OF than YF at all locations except the forehead and hand the forearm, hand, anterior thigh, posterior calf, and dorsal foot only $(\mathrm{p}<0.05)$. Whilst for males post hoc comparison indicated that thermal sensitivity was lower in the OM compared to YM at all locations except the forehead.

\subsection{Warm thermal sensitivity}

The absolute heat-flux thresholds for warm sensitivity of each body region in the four groups are shown in Figure 1. Three way-ANOVA revealed a main effect of age as the elderly group had a lower thermal sensitivity than the younger group $\left(2127.0 \pm 1208.3 \mathrm{~W} / \mathrm{m}^{2}\right.$ and $1301.7 \pm 1055.2 \mathrm{~W} / \mathrm{m}^{2}$, respectively, $\left.\mathrm{p}<0.001\right)$. A main effect of sex was also found as females had a higher thermal sensitivity in comparison to males $\left(683.4 \pm 314.4 \mathrm{~W} / \mathrm{m}^{2}\right.$ and $1211.3 \pm$ $567.6 \mathrm{~W} / \mathrm{m}^{2}$, respectively, $\left.\mathrm{p}<0.001\right)$. A main effect of location was observed and post hoc comparisons are presented in Table $3(\mathrm{p}<0.001)$. Inclusive of all ages and sex, high to low 
regional thermal sensitivity was in the following order: forehead $\left(906.4 \pm 508.9 \mathrm{~W} / \mathrm{m}^{2}\right)$, anterior forearm $\left(1148.6 \pm 523.1 \mathrm{~W} / \mathrm{m}^{2}\right)$, dorsal hand $\left(1309.6 \pm 825.4 \mathrm{~W} / \mathrm{m}^{2}\right)$, chest $(1462.1 \pm$ $\left.621.1 \mathrm{~W} / \mathrm{m}^{2}\right)$, anterior thigh $\left(1506.1 \pm 734.8 \mathrm{~W} / \mathrm{m}^{2}\right)$, scapula $\left(1829.6 \pm 1064.1 \mathrm{~W} / \mathrm{m}^{2}\right)$, posterior calf $\left(2083.3 \pm 1131.3 \mathrm{~W} / \mathrm{m}^{2}\right)$ and dorsal foot $\left(2411.4 \pm 1335.4 \mathrm{~W} / \mathrm{m}^{2}\right)$. A significant interaction was observed between age and location and post hoc comparison revealed that all 8 body regions were significantly more sensitive in the younger group compared to the elderly group $(\mathrm{p}<0.05)$ except the forehead. An interaction was also observed and between sex and location and post hoc comparison revealed that all locations except the chest and calf were hand was more sensitive in females compared to males $(\mathrm{p}<0.05)$.

There was no interaction between age, sex and location $(\mathrm{p}>0.05)$ but when the effect of location and age was analysed for males and females separately, the results revealed a significant interaction between age and location. Post hoc comparison indicated that thermal sensitivity was higher in YF than OF at all locations except the forehead $(p<0.05)$. Whilst for males post hoc comparison indicated that thermal sensitivity was lower in the OM compared to YM at all locations except the forehead, chest and back-and hand.

\subsection{Temperature differences}

The influence of age and sex on regional thermal sensitivity to either an increasing (warm) or decreasing (cold) stimulation was analysed using 4-way repeated measures ANOVA

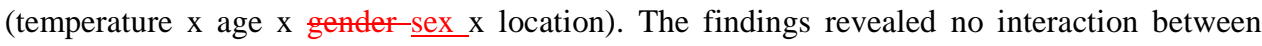
temperature, age, gender $\underline{\operatorname{sex}}$ and location $(\mathrm{p}>0.05)$. There was an interaction between temperature and sex as females had a lower warm and cold thermal sensitivity compared to males $(\mathrm{p}<0.05)$. There was an interaction between temperature and age as the younger adults 
had a lower warm and cold threshold compared to older participants $(\mathrm{p}<0.05)$. There was an interaction between temperature, location and age $(\mathrm{p}<0.05)$. Regional thermal sensitivity during the cold threshold tests was more homogenous for young and older participants however during warm threshold tests, regional thermal sensitivity showed more variation especially in the younger participants. The forehead sensitivity was similar between cold and warm threshold tests in both age groups, whilst the anterior forearm, posterior calf and dorsal foot were higher during warm and cold threshold testing for both age groups. There was no interaction between temperature, age and sex $(\mathrm{p}>0.05)$.

\subsection{Discussion}

The aim of the present study was to investigate the effect of ageing on regional warm and cold thermal sensitivity in males and females. The main findings of the present study were that thermal sensitivity decreases with aging in each sex and that regional differences exist in the age-related decrement of thermal sensitivity. Regional differences in thermal sensitivity are more homogenous for cold sensitivity in comparison to a more heterogeneous warm sensitivity and although sensitivity declines with ageing the regional differences are unchanged by ageing. Interestingly, whilst the thermal sensitivity declines with ageing, this decline appears to be attenuated on the forehead in both sexes. Finally, females demonstrated a greater thermal sensitivity (to both warm and cold) than males regardless of age.

\subsection{Effect of ageing and sex}

A considerable amount of research exists investigating the interface between the skin and the 
environment and its responsiveness to a variety of stimuli. Furthermore, research has shown that the sensitivity of the skin to such stimuli declines with ageing $[7,8,9,10,11]$. From the present study there is clear evidence that thermal sensitivity declines with ageing to both a cold (older: $1468.6 \pm 744.7 \mathrm{~W} /-\mathrm{m}^{2}$, younger: $\left.869.8 \pm 654.7 \mathrm{~W} /-\mathrm{m}^{2}, \mathrm{p}<0.001\right)$ and warm (older: $2127.0 \pm 1208.3 \mathrm{~W} /-\mathrm{m}^{2}$, younger: $\left.1301.7 \pm 1055.2 \mathrm{~W} / \mathrm{m}^{2}, \mathrm{p}<0.001\right)$ innocuous stimulus. Whilst the present study supports previous research, it also adds to our current knowledge about the decline in thermal sensitivity with ageing and how it differs between the two sexes. The results indicated that there was no interaction between age and sex as both male and female thermal sensitivity declined with ageing for a warm and a cold sensitivity. As observed with our previous findings in young participants [17], females displayed a greater thermal

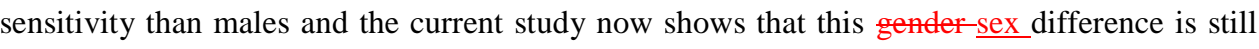
apparent in aged individuals (>65yrs). This suggests that the decline in sensitivity with ageing may be relative to the initial sensitivity of the individual or location. The exception to this rule is the forehead, which that will be discussed below.

Skin temperature is known to vary across the body and differ between males and females [25] but remain unchanged with age $[7,26]$. The current study utilised a heat flux technique, which reduces the influence of varying skin temperature on thermal sensitivity [27, 28, 29]. The actual mechanism for the decline in sensitivity with ageing is beyond the realms of this study by previous research may shed some light on this matter. Grassi et al. [30] exposed elderly $(71.6 \pm 2.2 \mathrm{yrs})$, middle aged $(46.2 \pm 1.9 \mathrm{yrs})$ and young $(23.8 \pm 1.8 \mathrm{yrs})$ participants to dynamic ambient temperatures to monitor muscle and skin sympathetic nerve activity. They noted that despite no differences in skin temperature, the elderly participants displayed 
progressive and marked reductions in skin sympathetic nerve activity compared to the middle aged and younger group. This suggests that the decline in sensitivity observed in the present study may be associated with an age-dependent neural degeneration. Grassi et al. [30] ruled out any impairment to efferent sympathetic neurons due to an unaltered SSNA response to an emotional stimulus amongst the three age groups. Therefore the age related decline in thermal sensitivity might occur within the afferent signals from peripheral thermoreceptors. There have been other findings in support of this hypothesis with evidence of reduced thermoreceptors density, decreased conduction velocities and/or neurotransmitter release (see review: Verdu et al. [1]). Alternatively, central integration in the hypothalamus may also contribute to the age dependent decline in thermal sensitivity but further research is required to clarify this.

In the present study, the sex-related differences suggest that thermal sensitivity is more pronounced in female than that of male in each age group, which may suggest that females initiate a behavioral temperature regulation response earlier than males. It has been suggested that females are more sensitive than males to a thermal stimulus due to an impaired ability to thermoregulate in comparison to male counterparts [17, 31]. Therefore, it would be beneficial for females to be more sensitive to a warm or cold stimulus than males in order to encourage behavioural responses to maintain thermal balance. The present study clearly indicates that females are still more sensitive than males regardless of age. The interaction between age and gender $\underline{\text { sex }}$ on cutaneous vascular and sudomotor function is currently unknown. The current data may suggest that the age related decline typically observed is similar between males and females but further research is required to investigate this. However, if males cutaneous vascular and sudomotor function responses to dynamic ambient conditions is impaired more so 
than females, this alongside lowered thermal sensitivity puts them at a greater risk for thermal imbalance.

It is noteworthy to highlight that whilst there were differences in subcutaneous fat (as measured by skin fold analysis) between sexes and ages amongst the female group we do not believe that this will have contributed to the differences observed. Whilst body fat and/or skin fold thickness can impact autonomic thermoregulation there is limited evidence for its role in perceptual thermal sensitivity. It has consistently been reported that participants with more subcutaneous fat tend to have a lower skin temperature [32, 33, 34]. Our findings agree with this as our OF had a higher $\Sigma 7 \mathrm{SF}$ and a lower mean skin temperature, whilst our males demonstrated no differences in $\Sigma 7 \mathrm{SF}$ or skin temperature. However, there is very limited evidence to show that skin fold thickness or body fat affects thermal sensitivity. Budd et al. [35] found that whilst fatter participants differed in skin temperature and core temperature they felt no less uncomfortable to a whole body cold exposure test compared to leaner individuals. This has recently been supported by Fournet [36], who found that despite colder skin temperatures, fatter participants had similar local thermal sensations as their leaner counterparts. Ouzzahra [37] also found no relationship between thermal sensation and skin fold thickness across numerous sites on the body. A reason for this finding maybe that the thermoreceptors being stimulated, along with other sensory receptors, are located in the dermis of the skin. Subcutaneous fat on the other hand, sits below the dermis in the hypodermis where neither cold nor warm thermoreceptors lie. The reason autonomic response may be influenced by subcutaneous fat is that the sweat glands and cutaneous blood vessels are located in the hypodermis, which is the site of adipose tissue. Notably, cold and warm receptors have been found at different depths within the dermis and this has been suggested as one of the reasons for a heightened sensitivity to cold compared to a warm $[50,51,52,46]$. This concept is discussed in 
Section 4.3 below.

It is possible that the skin thickness may explain the aging differences observed in our study, as

there is some evidence to suggest skin thickness declines with age [38]. However, our methods

do not accurately measure skin thickness as skin fold calipers combines both subcutaneous fat and

skin thickness, with subcutaneous fat contributing more substantially to this measure. Evidently,

further research on this topic is warranted.

\subsection{Effect of ageing and regional differences}

Regional differences in thermal sensitivity to a warm and cold stimulus were apparent in both age groups and the data indicated that all 8 body regions were more sensitive in the younger group compared to the elderly group $(\mathrm{p}<0.05)$. The pattern of sensitivity across the body depends on the type of stimulus (hot or cold) and the age (young or elderly). However what is very clear from the data is that the forehead was consistently the most sensitive region in both age groups, both sexes and to either an increasing or decreasing stimulus. In addition, whilst thermal sensitivity declines with ageing in all locations the thermal sensitivity at the forehead was attenuated in comparison to the other locations. Cotter and Taylor [3ㄹ] demonstrated that the face is a highly thermosensitive area for both perceptual thermal sensitivity and autonomic sensitivity (i.e. influence on sudomotor control) in comparison to numerous locations across the body. In addition, Gerrett et al. [16, 17] showed that thermal sensitivity across 31 locations on the body declines during exercise in comparison to rest but this decline is not observed at the forehead to a warm or cold stimulus in females. Collectively this data suggest that the thermal sensitivity of the face must be of 
physiological importance. By thermally or non thermally stimulating the face using convective heating or cooling or topical application of menthol or capsicum (respectively), Schlader et al. [4033] demonstrated that under neutral conditions $\left(20.3 \pm 0.2^{\circ} \mathrm{C}, 48 \pm 3 \% \mathrm{RH}\right)$ the face is capable of modulating thermoregulatory behavior during exercise. Although no data is available about the role of facial thermal sensitivity on thermoregulatory behavior during rest or in older individuals we speculate that thermal sensitivity is persevered at the forehead to maintain behavioural thermoregulation throughout the life span. Whether this preservation is a natural defence mechanism or a consequence of nurtured human behavior given that the face is typical uncovered by clothing in every day life remains unknown.

In the present study, the foot was the least sensitive region in both sexes and ages during an increasing stimulus only. It has been demonstrated that the length or peripheral nerves can influence sensitivity to tactile responses such as vibration perception [4134, 4235]. However if this were to be true for thermal sensitivity one would expect to see a proximal to distal decline in thermal sensitivity across the body. Whilst cold thermal sensitivity was more heterogeneous across the body, warm sensitivity was homogenous, with thermal sensitivity decreasing in the following order: forehead $\left(906.4 \pm 508.9 \mathrm{~W} / \mathrm{m}^{2}\right)$, anterior forearm $\left(1148.6 \pm 523.1 \mathrm{~W} / \mathrm{m}^{2}\right)$, dorsal hand $\left(1309.6 \pm 825.4 \mathrm{~W} / \mathrm{m}^{2}\right)$, chest $\left(1462.1 \pm 621.1 \mathrm{~W} / \mathrm{m}^{2}\right)$, anterior thigh $(1506.1 \pm$ $\left.734.8 \mathrm{~W} / \mathrm{m}^{2}\right)$, scapula $\left(1829.6 \pm 1064.1 \mathrm{~W} / \mathrm{m}^{2}\right)$, posterior calf $\left(2083.3 \pm 1131.3 \mathrm{~W} / \mathrm{m}^{2}\right)$ and dorsal foot $\left(2411.4 \pm 1335.4 \mathrm{~W} / \mathrm{m}^{2}\right)$. Although the head was the most sensitive and the feet the least sensitive, there was no clear proximal to distal pattern observed at the remaining locations. Interestingly, longitudinal data collected on elderly males from a number of experiments [see review; [436]) suggested that has demonstrated that cutaneous vasodilation- 
(CVD); sweat gland output (SGO) and heat activated sweat glands (HASG) decline with-

ageing but in a non uniform rate across the body. Inoue et al. [36] hypothesize that that the decrement in heat loss effector function (cutaneous vasodilation CVD; sweat gland output

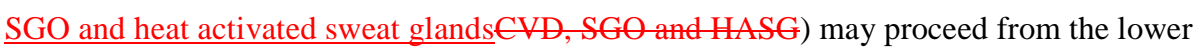
limbs, to the posterior torso, then the anterior torso, then the upper limbs and finally the head, which somewhat mimics the findings in the present study. This possible interaction between heat loss effector functions and thermal perceptions, if it exists, would be an interesting future research topic.

Using magnitude estimation both Gerrett et al. [16,17] and Nakamura et al. [4437] reported thermal sensitivity was greatest at the head, then the torso and declined towards the extremities (in relatively young participants). It has been suggested that regional sensitivity demonstrating this pattern is associated with protecting the vital organs located within the head and torso [4437]. However, thermal sensitivity using threshold detection technique does not support this theory but this may be associated with the different methodologies used. Alternatively, it has been suggested that the afferent fibres from the face are thought to ascend and terminate at different locations within the central nervous system than the free nerve endings from other locations $[4538, \underline{46} 39]$. In addition, animal studies have shown afferent convergent across the body but minimal convergence at the face $[4 \underline{7 \theta}, 4 \underline{8} 1]$. Although human data does not exist it is possible that the apparent sensitivity of the face yet the lack of clarity over other body sites observed in the present study is associated with the convergence onto common neurons.

\subsection{Effect of ageing and temperature}


In relatively young or middle-aged participants, it has been previously reported that humans are more sensitive to a cold stimulus in comparison to a warm stimulus using either threshold detection, method of limits or magnitude estimation $[16,4 \underline{\underline{q}} z, 19]$. This study supports these findings and also indicates that this is still apparent in aged individuals. Interestingly, in both age groups, thermal sensitivity was more homogenous for cold sensitivity and more heterogeneous to a warm stimulus and although sensitivity declines with ageing the regional differences are unchanged by ageing. Peripheral cold receptors are believed to be more superficial than warm receptors, greater in number and conduct at faster velocities due to being located on myelinated afferent fibres $[50,51,52,4643,44,45,39]$, which may explain the heightened sensitivity to cold compared to warmth. As there was no interaction between temperature, age and sex suggests that the decline in warm and cold sensitivity that occurs with ageing is not different between males or females. That is, thermal sensitivity will decline in a similar manner between males and females with the ageing process. In contrast, Fowler et al. [5346] reported greater increases in the warm threshold detection (i.e. sensitivity declined) in comparison to cold threshold detection in aged individuals; suggesting that the unmyelinated fibres were age affected. Differences may be due to the methods as they used an increasing stimulus temperature of $1.0^{\circ} \mathrm{C} / \mathrm{s}$ and repeated stimulation of the skin to determine the thermal threshold, which differs to that studied here.

\subsection{Conclusion}

In summary, we found that thermal sensitivity to an increasing or decreasing temperature declines with ageing. Interestingly females demonstrated a greater thermal 
sensitivity (to both warm and cold) than males regardless of age. And whilst females are more sensitive, the decline that occurs with ageing is similar between the two genderssexes. Finally, whilst the thermal sensitivity declines with ageing, this decline appears to be attenuated on the forehead in both sexes 


\subsection{References}

[1] E. Verdú, D. Ceballos, J.J. Vilches, X. Navarro,-(2002). Influence of aging on peripheral nerve function and regeneration. J. Peripher. Nerv. Syst. 15 (2002) 191-208.

[2] C.M. Smith, L.M. Alexander, L. Kenney, Non-uniform, age related decrements in regional sweating and skin blood flow. Am. J. Physiol. Regul. Integr. Comp. Physiol, 35 (2013),-8, R877-85.

[3] Y. Inoue, Longitudinal effects of aging on heat-activated sweat density and output in healthy active older men. Eur. J. Appl. Physiol 74 (1996) 72-77.

[4] Y. Inoue, M. Shibasaki, Regional differences in age-related decrements of the cutaneous vascular and sweating responses to passive heating. Eur. J. Appl. Physiol 74 (1996) 78-84.

[5] G.S. Anderson, G.S. Meneilly, I.B. Mekjavic, Passive temperature lability in the elderly. Eur. J. Appl. Physiol. 73 (1996) 278-286.

[6] D.W. DeGroot, W.L Kenney, Impaired defense of core temperature in aged humans during mild cold stress. Am. J. Physiol. Regul. Integr. Comp. Physiol. 292 (2007) R103-R108.

[7] Y. Tochihara, T. Kumamoto, J-Y. Lee, N. Hashiguchi, Age-related differences in cutaneous warm sensation threshold if human males in thermoneutral and cool environments.

J. Therm. Biol. 36 (2011) 105-111.

[8] H-W. Huang, W-C. Wang, C-C-K. Lin, Influence of age on thermal thresholds, thermal pain thresholds and reaction time. J. Clin. Neurosci. 17 (2010) 722-726.

[9] G. Pickering, D. Jourdan, A. Eschalier, C. Dubray, Impact of age, gender and cognitive functioning on pain perception. Gerontol. 48 (2002) 112-118.

[10] S. Lautenbacher, M. Kunz, P. Strate, J. Nielsen, L. Arendt-Nielsen, Age effects of pain 
thresholds, temporal summation and spatial summation of heat and pPressure pain. Pain. 115 (2005) 410-418

[11] J.C. Stevens, M. Alvarez-Reeves, L. Dipletro, G.S. Mack, B.G. Green, Decline of tactile acuity in aging: a study of body site, blood flow, and lifetime habits of smoking and physical activity. Somatosens. Mot. Res. 20, (2003) 271-279.

[12] D.R.Sr, Kenshalo, Somesthetic sensitivity in young and elderly humans. J. Gerontol. 41 (1986) 732-742.

[13] E-L. Haruj, Cold and warmth perception mapped for age, gender and body area. Somatosens. Mot. Res. 19 (2002) 61-75.

[14] H. Strughold, R. Porz, Die dichte der kaltpunkte auf der haut des mens- chlichen korpers, Z. Biol. 91 (1931) 563-571.

[15] H.Rein, Über die topographie der warmempfindung. beziehungen zwischen innervation und rezeptorischen endorganen. Z. Biol. 82 (1925) 513-535.

[16] N. Gerrett, Y. Ouzzahra, B. Redortier, T. Voelcker, G. Havenith, Female thermal sensitivity to hot and cold during rest and exercise. Physiol. Behav. 152 (2015) 11-19.

[17] N. Gerrett, Y, Ouzzahra, S. Coleby, S. Hobbs, B. Redortier, T. Voelcker, G. Havenith, Thermal sensitivity to warmth during rest and exercise. A sex comparison. Eur. J. Appl. Physiol. 114 (2014) 1451-62.

[18] Y. Ouzzahra, G. Havenith, B. Redortier, Regional distribution of thermal sensitivity to cold at rest and during mild exercise in males $_{-,}$, J. Therm. Biol. 37 (2014) 517-523. doi:

10.1016/j.jtherbio.2012.06.003 
[19] P. Golja, M.J. Tipton, I.B. Mekjavic, Cutaneous thermal thresholds--the reproducibility of their measurements and the effect of gender, J. Therm. Biol. 28 (2003) 341-346.

[20] S. Lautenbacher F, Strian F Sex differences in pain and thermal sensitivity: the role of body size. Percept. Psychophys. 50 (1991) 179-183. doi: 10.3758/BF03212218

[21] R.B. Fillingim, W. Maixner, S. Kincaid, S. Silva, Sex differences in temporal summation but not sensory-discriminative processing of thermal pain, Pain. 75 (1998) 121-127. doi:

$10.1016 / \mathrm{S} 0304-3959(97) 00214-5$

[22] S. Fujimoto, T. Watanabe, Studies on the body surface area of Japanese $e_{2}-$ Acta. Med. Nagasaki. 13 (1969) 1-13.

[23] B.A. Franklin, M.H. Whaley, E.T. Howley, G.T. Balady, ACSM's guidelines for exercise testing and prescription. Lippincott Williams \& Wilkins, Philadelphia, USA, 2000.

[24] N.L. Ramanathan, A new weighting system for mean surface temperature of the human body, J. Appl. Physiol. 19 (1964) 531-533.

[25] D. Fournet, L. Ross, T. Voelcker, B. Redortier, G. Havenith, Body mapping of thermoregulatory and perceptual responses of males and females running in the cold, J. Therm. Biol. 38 (2013) 339-344. doi: 10.1016/j.jtherbio.2013.04.005

[26] Y. Inoue, M. Shibasaki, K. Hirata, T. Araki, Relationship between skin blood flow and sweating rate and age related regional differences. Eur. J. Appl. Physiol, 79 (1998) 17-23.

[27] R. Ihno, T. Tamura, K. Oda, The trial of the measurement of thermal threshold of the human body. Jpn. J. Physiol. Anthropol., 4 (1999) 1-13.. 
[28] S. Jinno, Useful methods for the general clinicians to cope with the patients' paralysis and pain. The Nippon Dental Review 11 (2004) 135

[29] S. Jinno, Y. Kabasawa, Y. Satoh, The evaluation of thermal threshold on the facial region-the efficacy of measurement using the heat flux technique. J. Jpn. Dent. Soc. Anesthesiol. 34 (2006) 492-497.

[30] G. Grassi, G. Seravell, C. Turri, G. Bertinieri, R. Dell’Oro, G. Mancia, Impairment of thermoregulatory control of skin sympathetic nerve traffic in the elderly. Circulation. 108 (2003) 729-735.

[31] Y. Inoue, Y. Tanaka, K. Omori, T. Kuwahara, Y. Ogura, H. \& Ueda, Sex- and menstrual cycle--related differences in sweating and cutaneous blood flow in response to passive heat exposure. Eur. J. Appl. Physiol. 94 (2005) 323-332.

[32] J. LeBlanc. Subcutaneous fat and skin temperature. Can. J. Biochem. Cell. B. 32 (1954), 354-358.

[33] J. Frim, S.D. Livingstone, L.D. Reed, R.W. Nolan, R. E. Limmer, Body composition and skin temperature variation. J. Appl Physiol. 68 (1990) 540-.543

[34] A.M.J. Claessens-van Ooijen, K.R. Westerterp, L. Wouters, P.F.M. Schoffelen, A.A. van $\underline{\text { Steenhoven, W.D. van Marken Lichtenbelt, Heat production and body temperature during }}$ cooling and rewarming in overweight and lean men. Obesity, 14 (2006), 1914-1920. 
[35] G.M. Budd, J.R. Brotherhood, A.L. Hendrie, S.E. Jeffrey. Effect of fitness, fatness, and age on men's response to whole body cooling in air. J. Appl. Physiol. 71 (1991) 2387-2393.

[36] D.Fournet. Skin temperature variations in the cold [PhD Thesis]. Loughborough University, UK. 2013.

[37] Y. Ouzzahra. Regional thermal sensitivity to cold at rest and during exercise. [PhD

Thesis]. Loughborough University, UK, 2012.

[38] J.M. Weller, H.I. Maibach. Age and skin structure and function a quantitative approach (I): blood flow, pH, thickness, and ultrasound echogenicity. Skin Res. Technol. 11 (2005) 221-235.

[39z] J.D. Cotter, N.A. Taylor, The distribution of cutaneous sudomotor and alliesthesial thermosensitivity in mildly heat-stressed humans: an open-loop approach. J Physiol. 565

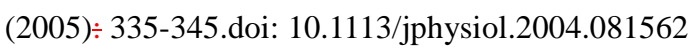

[4033] Z.J. Schlader, S.E. Simmons, S.R. Stannard, T. Mundel, The independent roles of temperature and thermal perception in the control of human thermoregulatory behavior. Physiol. Behav. 103 (2011) 217-224. doi: 10.1016/j.physbeh.2011.02.002

[4134] N. Desphande, J. Metter, S. Ling, R. Conwit, L. Ferrucci, Physiological correlates of age-related decline in vibrotactile sensitivity institution of origin .Neurobiol.Aging. 29 (2008) 765-773. 
[4235] F. Gerr, R. Letz, Covariates of human peripheral nerve function: II. Vibrotactile and thermal thresholds. Neurotoxicol. Teratol., 16 (1994) 105-112.

[4336] Y. Inoue, T. Kuwahara, T. Araki, Maturation- and ageing-related changes in heat loss effector function. J. Physiol. Anthropol. Appl. Hum. Sci. 23 (2004) 289-294

[4437] M. Nakamura, T. Yoda, L.I. Crawshaw, S. Yasuhara, Y. Saito, M. Kasuga, et al., Regional differences in temperature sensation and thermal comfort in humans, J. Appl.

Physiol. 105 (2008) 1897-1906. doi: 10.1152/japplphysiol.90466.2008

[4538] H.D. Schwark, C.G. Tennison, O.B. Ilynsky, Influence of skin temperature on cuneate neuron activity. Soc. Neurosci. Abstracts. 23 (1997), 2340.

[4639] H. Hensel, Thermoreception and temperature regulation, Monogr. Physiol. Soc. 38 (1981) 1-31

[4그] R.F. Hellon, D. Mitchell, Convergence in a thermal afferent pathway in the rat. J. Physiol, 289 (1975) 57-58.

[4ㅌt] A. Iggo, R.L. Ramsey, Thermosensory mechanisms in the spinal cord of monkeys. In Sensory Functions of the skin in Primates with special reference to Man, ed. Zotterman, Y., pp285-304. Pergamon Press, Oxford, UK. 1976

[4ㅁz] J.Y. Lee, M. Saat, C. Chou, N. Hashiguchi, T. Wijayanto, H. Wakabayashi, et al., Cutaneous warm and cool sensation thresholds and the inter-threshold zone in Malaysian and Japanese males, J. Therm. Biol. 35 (2010) 70-76.

[5043] Y. Zotterman, Special senses: Thermal receptors. Annu. Rev. Physiol. 15 (1953) 357-372. 
[5144] A.C. Guyton, Textbook of Medical Physiology. W.B. Saunders, Philadelphia, USA, 1991

[5245] H. Hensel, K. H. Andres, M. Düring, Structure and function of cold receptors, Pfügers Archiv. 352 (1984) 1-10.

[5346] C.J. Fowler, M. Carroll, D. Burns, N. Howe, K. Robinson, A portable system for measuring cutaneous thresholds for warming and cooling, J. Neurol. Neurosur. PS. 50 (1978) 1211-1215. doi: 10.1136/jnnp.50.9.1211 


\section{Acknowledgments}

We thank our volunteer subjects-for participating in this study. This study was

partly supported by a Grant-in-Aid for Scientific Research (grant nos. 16500435 and 20247035). 


\section{Figure Legends}

Figure 1. The absolute heat-flux threshold (warm and cold thermal sensitivity) of each body region in older female (OF) and younger female (YF) participants subjects-and older male $(\mathrm{OM})$ and younger male $(\mathrm{YM})$ subjectsparticipants. Values are means $\pm \mathrm{SEM}$.

* indicates locations where younger participants were more sensitive than older participantsSignificantly different between older and younger subjects for each sex, $P<0.05$.

$\dagger$ indicates locations where female participants were more sensitive than male participantsSignificantly different between female and male subjects for each age, $P<0.05$.

* Significantly different between warm and cold thermal sensitivity, $P<0.05$. 
Table 1. Physical characteristics of each group.

\begin{tabular}{|c|c|c|c|c|c|c|c|c|}
\hline & $\mathbf{n}$ & $\begin{array}{l}\text { Age } \\
(y r)\end{array}$ & $\begin{array}{c}\text { Height }^{\#} \\
(\mathrm{~cm})\end{array}$ & $\begin{array}{l}\text { Mass }^{\#} \\
(\mathrm{~kg})\end{array}$ & $\begin{array}{l}\text { AD/mass }{ }^{\#} \\
\left(\mathrm{~cm}^{2} / \mathrm{kg}\right)\end{array}$ & $\begin{array}{l}\mathbf{\Sigma 7 S F}^{\#} \\
(\mathrm{~mm})\end{array}$ & $\begin{array}{c}\mathbf{V O} \mathbf{O}_{2} \mathbf{m a x} \\
(\mathrm{ml} / \mathrm{kg} / \mathrm{min})\end{array}$ & $\begin{array}{c}\text { PA } \\
\text { (steps/day) }\end{array}$ \\
\hline Older female & 12 & $65.2 \pm 1.0 *$ & $152.8 \pm 1.8 *{ }^{\dagger}$ & $51.5 \pm 2.5^{\dagger}$ & $281.1 \pm 6.4$ & $148 \pm 10 *, \dagger$ & $33.8 \pm 1.9 *$ & $11650 \pm 1112$ \\
\hline Younger female & 29 & $21.6 \pm 0.2$ & $161.0 \pm 1.1^{\dagger}$ & $53.2 \pm 1.1^{\dagger}$ & $284.5 \pm 2.7$ & $122 \pm 6^{\dagger}$ & $41.7 \pm 1.4$ & $12128 \pm 787^{\dagger}$ \\
\hline Older male & 17 & $66.2 \pm 1.5 *$ & $163.2 \pm 1.8 *$ & $59.0 \pm 2.1$ & $271.8 \pm 4.3$ & $69 \pm 5$ & $38.5 \pm 1.6$ & $11977 \pm 1177$ \\
\hline Younger male & 13 & $21.2 \pm 0.4$ & $171.2 \pm 1.2$ & $58.5 \pm 1.8$ & $281.0 \pm 4.3$ & $56 \pm 7$ & $43.3 \pm 3.1$ & $15801 \pm 1693$ \\
\hline
\end{tabular}

Values are means \pm SEM. AD: body surface area, $\Sigma 7 \mathrm{SF}$ : sum of 7 skinfolds, $\mathrm{VO}_{2}$ max: maximal oxygen uptake, PR: physical activity level.

* Significantly different between older and younger subjects-participants for each sex, $P<0.05$.

${ }^{\dagger}$ Significantly different between female and male participants subjects for each age, $P<0.05$.

${ }^{\#}$ Significantly different between female and males inclusive of all ages, $\mathrm{P}<0.05$ 
Table 2. Baseline oral $\left(\mathrm{T}_{\mathrm{or}}\right)$, mean skin $\left(\mathrm{T}_{\mathrm{sl}}\right)$ and mean body $\left(\mathrm{T}_{\mathrm{b}}\right)$ temperatures.

\begin{tabular}{lccc}
\hline & $\mathbf{T}_{\text {or }}$ & $\mathbf{T}_{\text {sk }}$ & $\mathbf{T}_{\mathbf{b}}$ \\
& $\left({ }^{\circ} \mathrm{C}\right)$ & $\left({ }^{\circ} \mathrm{C}\right)$ & $\left({ }^{\circ} \mathrm{C}\right)$ \\
\hline Older female & $36.73 \pm 0.06$ & $32.10 \pm 0.58 *$ & $36.26 \pm 0.10$ \\
Younger female & $36.71 \pm 0.05$ & $33.13 \pm 0.06$ & $36.36 \pm 0.05$ \\
Older male & $36.65 \pm 0.05$ & $32.98 \pm 0.13$ & $36.28 \pm 0.05$ \\
Younger male & $36.65 \pm 0.07$ & $33.11 \pm 0.18$ & $36.30 \pm 0.06$ \\
\hline
\end{tabular}

Values are means \pm SEM.

* Significantly different between older and younger participants subjects for each sex, $P<0.05$. 
Table 3. Statistical findings from pairwise comparison of each location for cold $(* \mathrm{p}<0.05, * * \mathrm{p}<0.001)$ and warm $(\dagger \mathrm{p}<0.05$, $\dagger \mathrm{p}<0.001)$ thermal sensitivity, inclusive of both sexes and ages. $(\mathrm{P}=$ posterior, $\mathrm{D}=$ dorsal, $\mathrm{A}=$ anterior $)$

\begin{tabular}{|c|c|c|c|c|c|c|c|c|}
\hline & Forehead & Chest & Scapula & A. Forearm & D. hand & A. Thigh & P. Calf & D. Foot \\
\hline Forehead & & $* *+\dagger$ & $* *+\dagger$ & $* * \dagger \dagger$ & $* *+\dagger$ & $* *+\dagger$ & $* *+\dagger$ & $* *+\dagger$ \\
\hline Chest & $* * \dagger \dagger$ & & $* \dagger$ & $\dagger \dagger$ & $*$ & & $\dagger \dagger$ & $\dagger$ \\
\hline Scapula & $* * \dagger \dagger$ & $* \dagger$ & & $\dagger$ & $\dagger 1$ & & $* *$ & $\dagger$ \\
\hline A. Forearm & $* * \dagger \dagger$ & $\dagger$ & $\dagger$ & & $*$ & $\dagger$ & $* \dagger \dagger$ & $\dagger$ \\
\hline D. hand & $* *+\dagger$ & $* *$ & †t & $*$ & & $*+$ & $* *+\dagger$ & $* *+\dagger$ \\
\hline A. thigh & $* * \dagger \dagger$ & & & $\dagger$ & $*$ & & $*_{-\dagger}$ & $\dagger$ \\
\hline P. calf & $* * \dagger \dagger$ & $\dagger$ & ** & *十† & $* * \dagger \dagger$ & 梡 & & $\dot{\varphi}$ \\
\hline D. foot & $* * \dagger \dagger$ & $\dagger \dagger$ & $\dagger \dagger$ & $\dagger \dagger$ & $* *+\dagger$ & $\dagger \dagger$ & $\dagger$ & \\
\hline
\end{tabular}


Cold Sensitivity

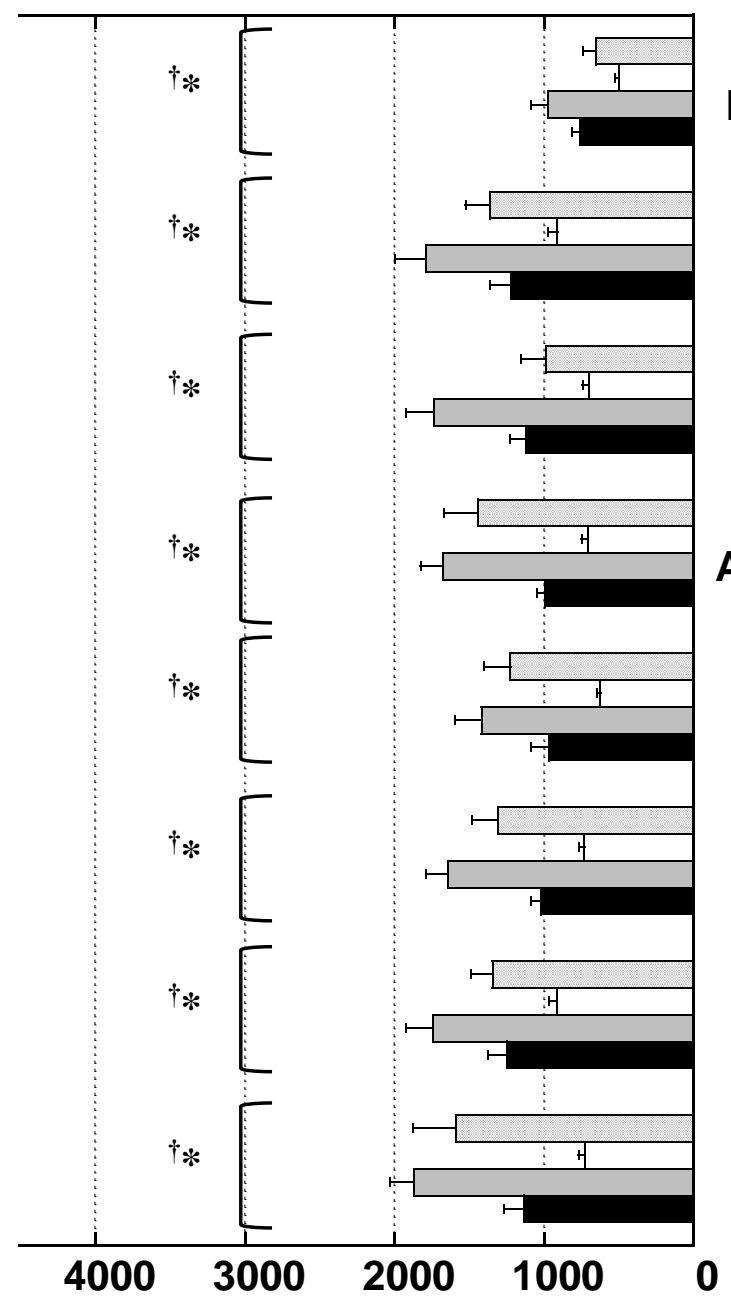

Warm Sensitivity

Forehead

Chest

Scapula

A.Forearm

D.Hand

A.Thigh

P.Calf

D.Foot

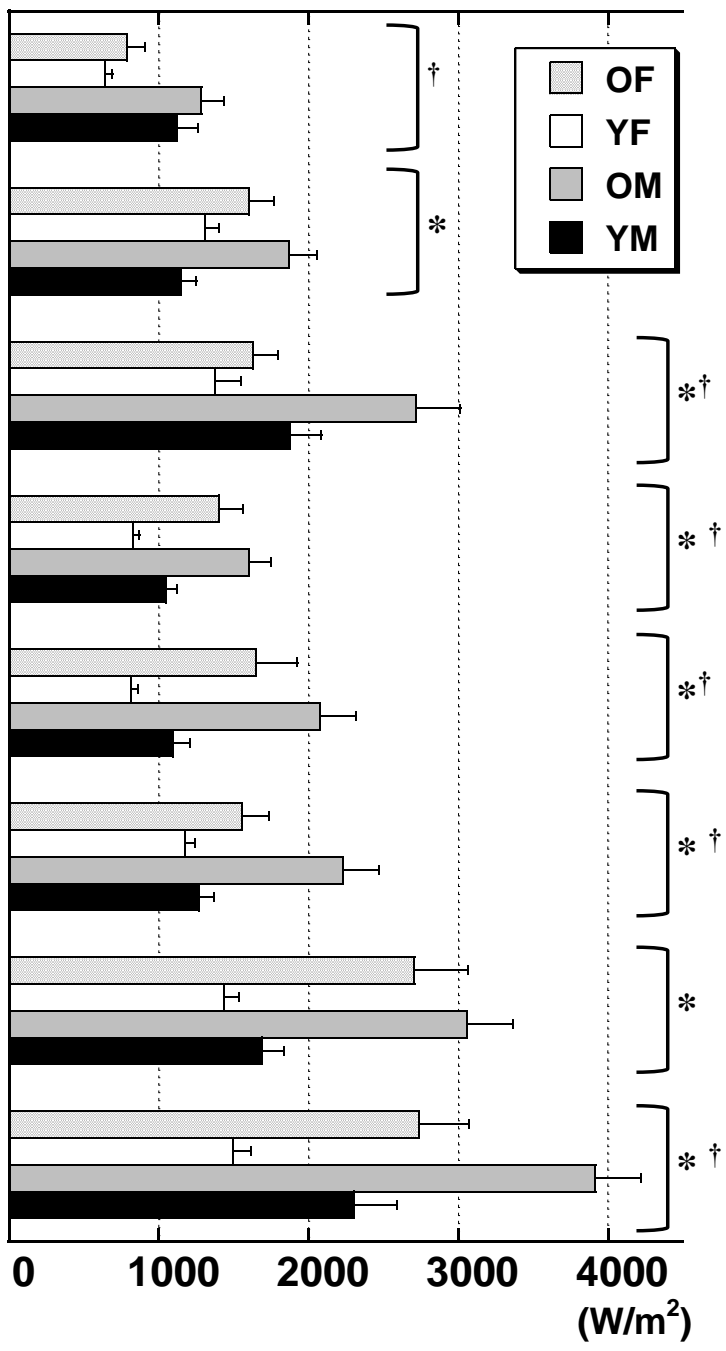

